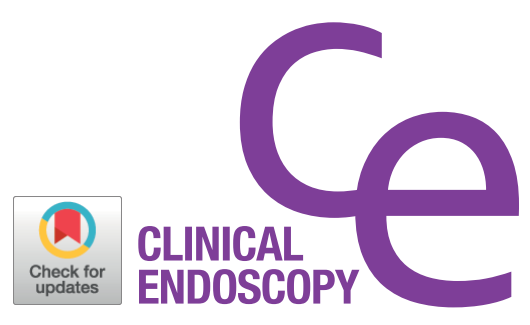

\title{
Hematochezia in Patient with Rectal Tumor: Consideration of Various Diagnostic Possibilities
}

\author{
Hae Min Jeong, Chang Seok Bang and Gwang Ho Baik \\ Department of Internal Medicine, Hallym University College of Medicine, Chuncheon, Korea
}

\section{Quiz}

A 74-year-old woman presented with hematochezia. The patient had bloody stool (approximately $50 \mathrm{cc}$ ) for the past 10 days. The patient had hypertension and was taking antihypertensive medications. The patient underwent total laparoscopic hysterectomy and bilateral salpingo-oophorectomy for endometrial cancer 2 years ago. After presentation, the patient underwent a colonoscopy. Approximately $3 \mathrm{~cm}$ mass was found near the anal canal (Fig. 1A). The mass was round and covered with exudate (Fig. 1B). Endoscopic ultrasonography showed a heterogeneous hypoechoic lesion located in the submucosal layer (Fig. 1C). Histopathological examination revealed small malignant oval-shaped cells with high nuclear-to-cytoplasmic ratio and poor differentiation (normal glandular structure was not observed) (Fig. 2). Computed tomography revealed a 3.5 $\mathrm{cm}$ enhancing lesion involving the distal rectum and anorectal junction (Fig. 3). Positron emission tomography revealed a mass of approximately $3-3.5 \mathrm{~cm}$ with increased FDG uptake $($ SUVmax $=10.79)$ in the distal rectum (Fig. 4). Transanal excision was performed, and the resected lesion showed an ill-demarcated ulcerative and fungating mass, measuring 3.3 $\times 2.7 \mathrm{~cm}$ extending to the pericolic soft tissue (Fig. 5). In the final pathological examination, round cells with a high nuclear-to-cytoplasmic ratio without macronucleoli, which invaded the entire mucosal layer, were observed (Fig. 2, 6A and B). Immunohistochemistry for CD-3 and CD-20 was negative. Diffuse brown colored pigmentation was observed (Fig. 6C), and immunohistochemistry for HMB- 45 and S-100 was positive (Fig. 7). What is the most probable diagnosis?

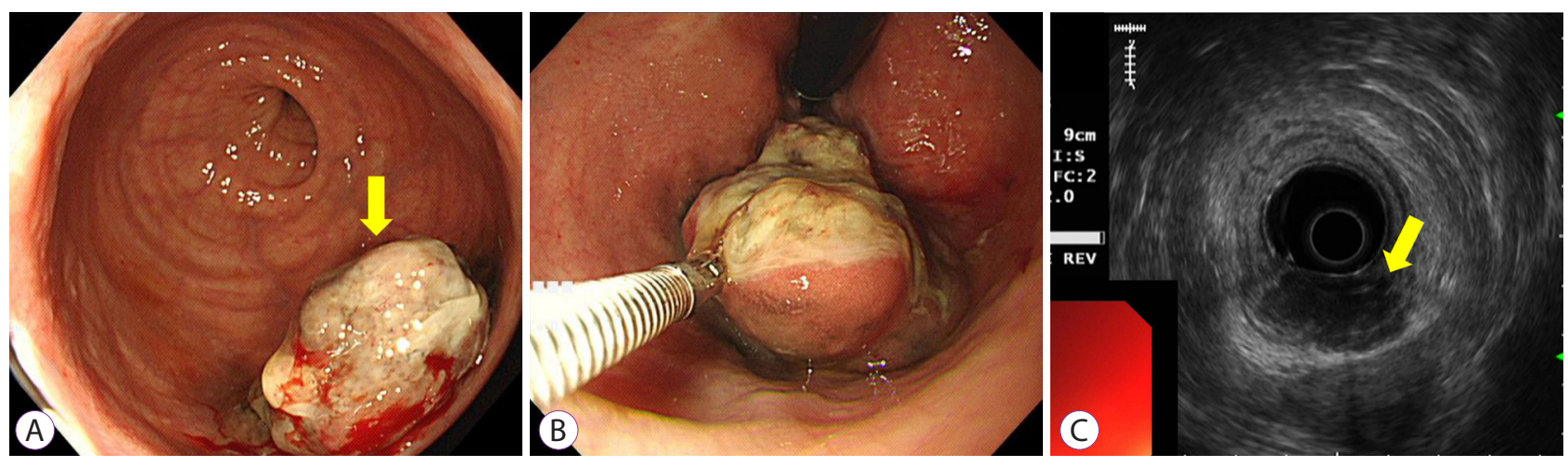

Fig. 1. (A, B) Colonoscopy demonstrating $3 \mathrm{~cm}$ mass covered with exudates near anal canal. (C) Endoscopic ultrasonography demonstrating heterogenous hypoechoic lesion in the submucosal layer.

Received: September 27, $2021 \quad$ Revised: October 7, 2021

Accepted: October 7, 2021

Correspondence: Chang Seok Bang

Department of Internal Medicine, Hallym University College of Medicine, 77 Sakju-ro, Chuncheon 24253, Korea

Tel: +82-33-240-5821, Fax: +82-33-241-8064, E-mail: csbang@hallym.ac.kr

ORCID: https://orcid.org/0000-0003-4908-5431

(c) This is an Open Access article distributed under the terms of the Creative Commons Attribution Non-Commercial License (http://creativecommons.org/licenses/by$\mathrm{nc} / 3.0$ ) which permits unrestricted non-commercial use, distribution, and reproduction in any medium, provided the original work is properly cited. 


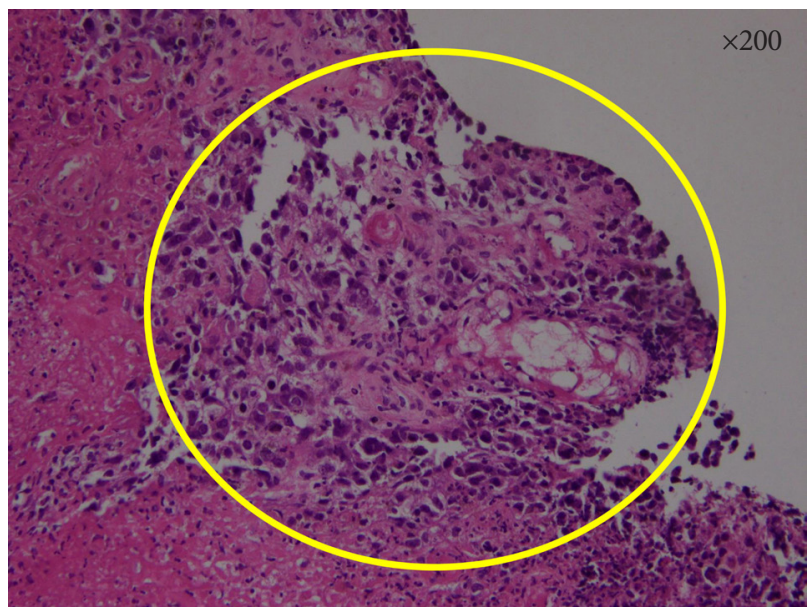

Fig. 2. Histopathological findings of the biopsy specimens. Small malignant oval-shaped cells with high nuclear-to-cytoplasmic ratio with poor differentiation (normal glandular structure was not observed) (hematoxylin and eosin stain $\times 200)$.

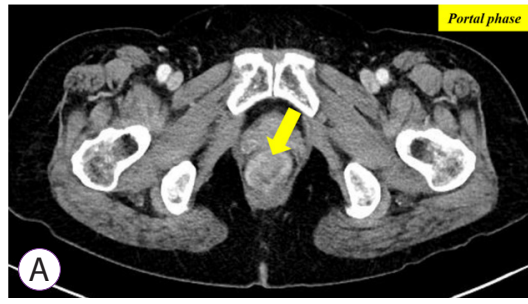

Fig. 3. (A, B) Contrast-enhanced computed tomography demonstrating $3.5 \mathrm{~cm}$ enhancing lesion involving distal rectum and anorectal junction.

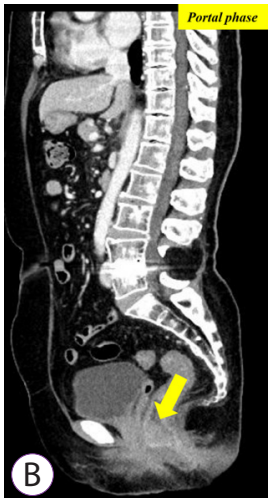

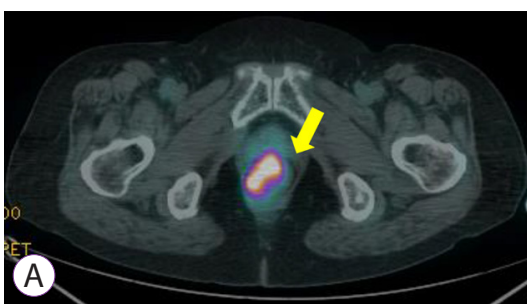

Fig. 4. (A, B) Positron emission tomography demonstrating 3-3.5 $\mathrm{cm}$ mass with increased FDG uptake (SUVmax $=10.79$ ) in the distal rectum.
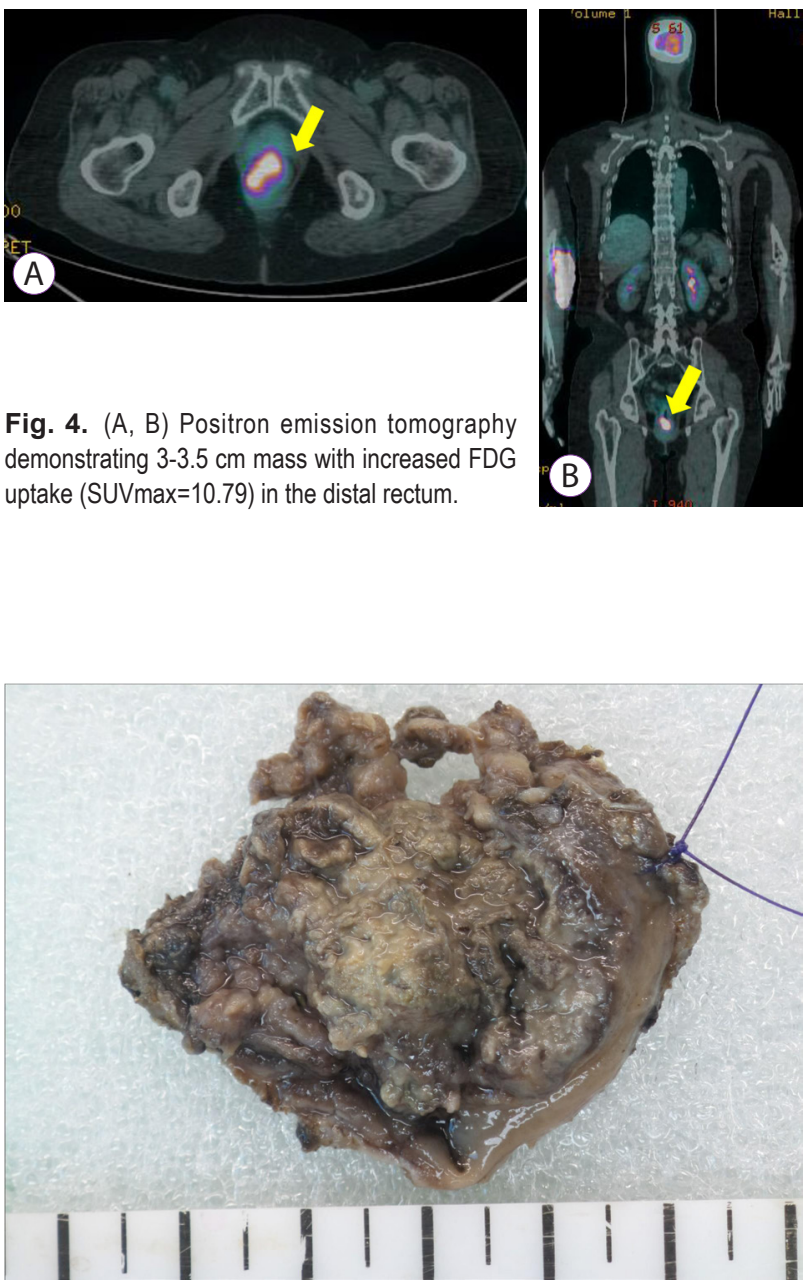

Fig. 5. Surgical specimen demonstrates an ill demarcated ulcerative and fungating mass, measuring $3.3 \times 2.7 \mathrm{~cm}$ extending to the pericolic soft tissue.
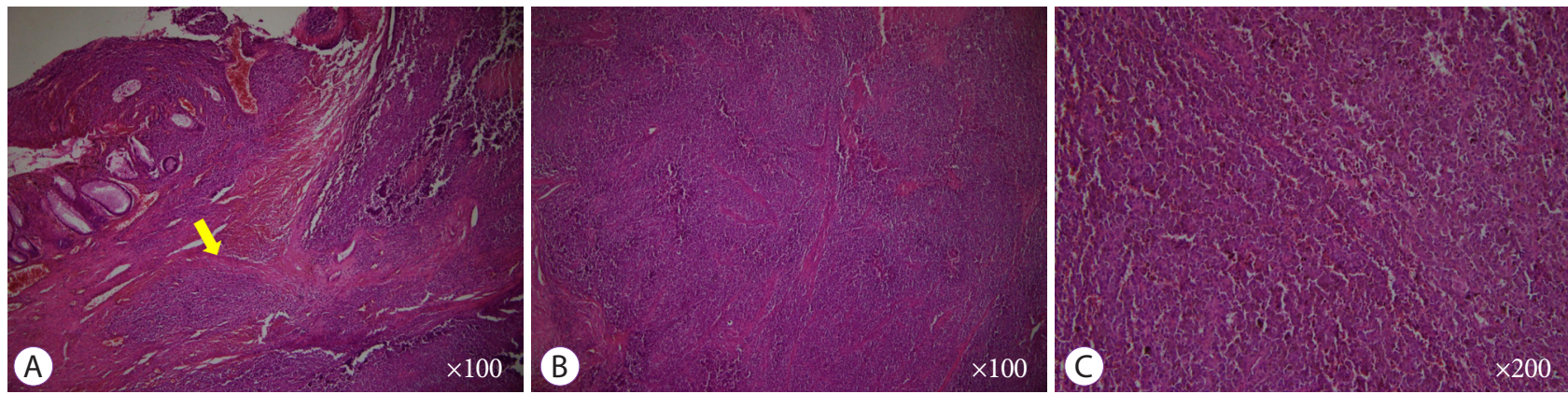

Fig. 6. Histopathological findings of the surgically resected specimen. (A, B) Round cells with a high nuclear-to-cytoplasmic ratio without macronucleoli which invaded whole mucosal layer (hematoxylin and eosin stain $\times 100$ ). (C) Diffuse brown colored pigmentation (hematoxylin and eosin stain $\times 200$ ). 

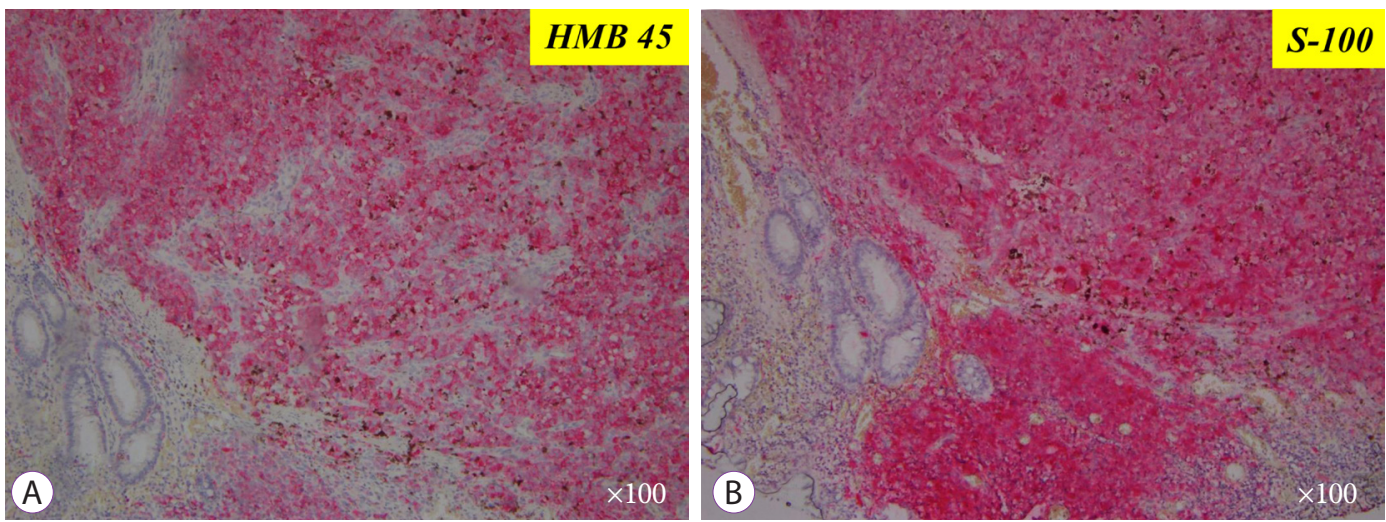

Fig. 7. Immunohistochemistry findings of the surgically resected specimen. (A, B) The tumor cells are diffusely positive for HMB-45 and S-100 (hematoxylin and eo$\sin \operatorname{stain} \times 100)$.

\section{Answer}

The patient was diagnosed with rectal melanoma. On colonoscopy, black pigmentation was not clearly observed on gross findings, and poorly differentiated adenocarcinoma was suspected based on the first histological examination. The mass was removed by transanal excision, and final immunohistochemistry for CK-20 (one of the markers for colorectal cancer) was negative, and for HMB-45 and S-100 was positive. Eventually, the patient was diagnosed with amelanotic melanoma. Primary anorectal melanoma is a rare tumor, accounting for only approximately $0.05 \%$ to $4.6 \%$ of all reported anorectal malignancies. ${ }^{1}$ Anorectal lesions account for $0.3 \%$ of all melanomas. ${ }^{2}$ Furthermore, approximately $30 \%$ of anorectal melanomas are amelanotic and can resemble benign polypoid lesions. ${ }^{3}$ The most common symptom is hemorrhage, ${ }^{1}$ and patients diagnosed with anorectal malignant melanoma usually have locoregional metastasis. ${ }^{4}$ Endoscopy or endoscopic ultrasound can be considered for diagnosis. ${ }^{5}$ Immunohistochemistry is the cornerstone for the diagnosis of malignant melanoma. S-100, HMB-45, vimentin, and Melan A are important markers for the diagnosis of malignant melanoma. There is currently no standard treatment for anorectal amelanotic melanoma due to the low incidence and lack of evidence in the literature. ${ }^{1}$ However, surgery in combination with immunotherapy, such as interferon, has been reported as a treatment option for localized tumors. ${ }^{6}$ Chemotherapy and radiation therapy are known to play only a limited role. Despite the recent treatment experience in Japan (successful treatment with nivolumab, an antibody to programmed death (PD-1) for controlling metastasis), ${ }^{8}$ the overall treatment experience is still limited. Therefore, early diagnosis is important for the prognosis.
Conflicts of Interest

The authors have no potential conflicts of interest.

Funding

None.

ORCID

Hae Min Jeong:

Chang Seok Bang:

Gwang Ho Baik:

https://orcid.org/0000-0003-0497-6732 https://orcid.org/0000-0003-4908-5431 https://orcid.org/0000-0003-1419-7484

\section{REFERENCES}

1. Xu X, Ge T, Wang G. Primary anorectal malignant melanoma: a case report. Medicine (Baltimore) 2020;99:e19028.

2. Chang AE, Karnell LH, Menck HR. The National Cancer Data Base report on cutaneous and noncutaneous melanoma: a summary of 84,836 cases from the past decade. The American College of Surgeons Commission on Cancer and the American Cancer Society. Cancer 1998;83:1664-1678.

3. Sahoo MR, Gowda MS, Kaladagi RM. Primary amelanotic melanoma of the rectum mimicking adenocarcinoma. Am J Case Rep 2013;14:280283.

4. Maqbool A, Lintner R, Bokhari A, Habib T, Rahman I, Rao BK. Anorectal melanoma--3 case reports and a review of the literature. Cutis 2004;73:409-413.

5. Stefanou A, Nalamati SPM. Anorectal melanoma. Clin Colon Rectal Surg 2011;24:171-6.

6. Kim KW, Ha HK, Kim AY, et al. Primary malignant melanoma of the rectum: CT findings in eight patients. Radiology 2004;232:181-186.

7. Malik A, Hull TL, Milsom J. Long-term survivor of anorectal melanoma: report of a case. Dis Colon Rectum 2002;45:1412-1415; discussion 1415-1417.

8. Tokuhara K, Nakatani K, Tanimura H, Yoshioka K, Kiyohara T, Kon M. A first reported case of metastatic anorectal amelanotic melanoma with a marked response to anti-PD-1 antibody nivolumab: a case report. Int J Surg Case Rep 2017;31:188-192.

9. Nafees R, Khan H, Ahmed S, Ahmed Samo K, Siraj Memon A. Primary rectal amelanotic malignant melanoma: a rare case report. Cureus 2020;12:e8115. 